\title{
Civilisations
}

Revue internationale d'anthropologie et de sciences

humaines

$41 \mid 1993$

Mélanges Pierre Salmon II

\section{Recherche sur l'identité ethnique du peuple Topoke (haut-zaire)}

Contribution à l'histoire socio-culturelle du Zaïre

\section{Bilusa Baila Boingaoli}

\section{OpenEdition}

Journals

Édition électronique

URL : http://journals.openedition.org/civilisations/1693

DOI : $10.4000 /$ civilisations. 1693

ISSN : 2032-0442

Éditeur

Institut de sociologie de l'Université Libre de Bruxelles

\section{Édition imprimée}

Date de publication : 1 septembre 1993

Pagination : 103-116

ISBN : 2-87263-094-5

ISSN : 0009-8140

Référence électronique

Bilusa Baila Boingaoli, « Recherche sur l'identité ethnique du peuple Topoke (haut-zaire) », Civilisations

[En ligne], 41 | 1993, mis en ligne le 28 juillet 2009, consulté le 19 avril 2019. URL : http://

journals.openedition.org/civilisations/1693; DOI : 10.4000/civilisations.1693

Ce document a été généré automatiquement le 19 avril 2019.

(c) Tous droits réservés 


\title{
Recherche sur l'identité ethnique du peuple Topoke (haut-zaire)
}

\author{
Contribution à l'histoire socio-culturelle du Zaïre
}

\author{
Bilusa Baila Boingaoli
}

\section{Introduction}

1 Maintes études ont déjà été réalisées sur les aspects politico-administratifs, économiques, démographiques et socio-culturels de l'organisation des sociétés africaines traditionnelles ainsi que sur le contact entre l'Afrique et les influences extérieures (esclavage et colonisation). Cependant, peu de chercheurs se sont penchés sur la dénomination réelle de certains groupes ethniques existant aujourd'hui. Presque toutes les monographies reprennent, souvent sans critique, les noms laissés par les premiers agents européens, alors qu'ils ne recouvrent pas la même importance, moins encore la même signification pour les populations qu'ils désignent.

2 Si certains groupes apparaissent plus étudiés et mieux connus, d'autres ne le sont que très imparfaitement. Biebuyck l'a d'ailleurs aussi constaté :

"Parmi la multitude de populations du Congo belge, il en existe qui, du point de vue ethnologique, sont actuellement bien connues... Il existe cependant une vaste aire occupée par une variété de populations et pour lesquelles nos renseignements restent très fragmentaires et insuffisants. Ce manque de connaissance est d'autant plus grave qu'il s'agit de groupes à très faible densité, dont la situation démographique n'est généralement pas florissante et dont la société est de plus en plus exposée à des influences nouvelles et à des modifications culturelles profondes" (Biebuyck, 1960 : 20-21).

3 Il fait allusion, dans sa note, aux Pygmées et aux Pygmol des (Bambuti de l'Ituri, Batswa de l'Equateur), aux Bakutu, aux Boyela et aux Ndengese de l'Equateur, aux Bakumu, aux Balese et aux Medje du Haut-Zaïre.

4 Cette observation mérite d'être étendue aux Topoke, l'un des principaux groupes ethniques du Haut-Zaïre et ayant une densité exceptionnellement élevée par rapport à la 
moyenne régionale $\left(14,61 \mathrm{hab} . / \mathrm{km}^{2}\right.$ chez les Topoke, contre $8,57 \mathrm{hab} . / \mathrm{km}^{2}$ pour la moyenne du Haut-Zaïre) (République du Zaïre, 1991 : 57-65). Ce peuple est connu, dans la littérature existante, sous plusieurs appellations : ESO, GESO, GESOGO, GESOHO, TOPOKE, TUPUKI, TOFORE, TUFUKI.

Ce bref exposé se propose de fournir des pistes susceptibles de contribuer à l'écriture de l'histoire des Topoke. Le premier effort, dans cette perspective, passe par la recherche de leur dénomination, élément si non essentiel, du moins fondamental d'identification. Sontils « ESO » ou « TOPOKE » ? D'où proviennent les éléments de contradiction? Quelle est la forme localement acceptée ? Cet exposé s'efforce de fournir des éléments de réponse à ces différentes questions.

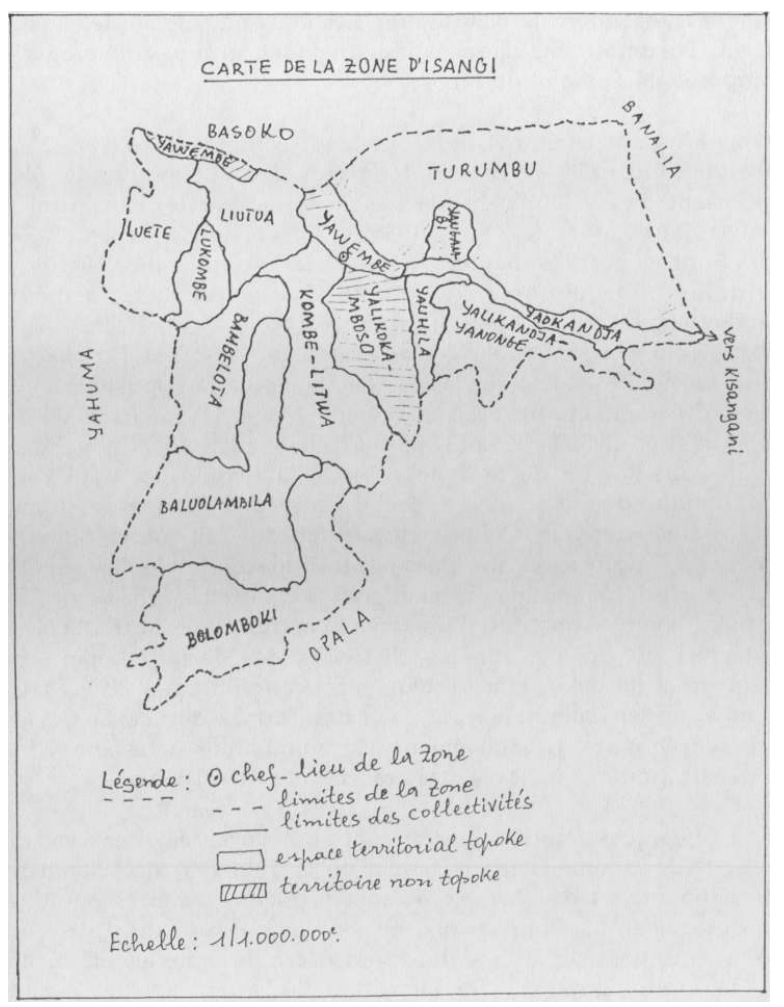

\section{Brève présentation des Topoke}

Les Topoke sont l'une des trois principales ethnies de la Zone d'Isangi (dans le HautZaïre). Les deux autres sont les Lokele et les Turumbu. Signalons aussi quelques groupes hétérogènes composés de Foma et de Mboso.

7 L'espace territorial des Topoke est compris entre $0^{\circ}$ et $2^{\circ}$ de latitude Sud, $23^{\circ}$ et $25^{\circ}$ de longitude Est. Le noyau le plus important de ce peuple habite l'angle formé par le Lomami et le fleuve Zaïre (Maes \& Boone, 1935 : 322. Voir aussi Bryan, 1959 : 46). Sont concernés par cet habitat, les Topoke des chefferies Baluolambila, Bolomboki, Kombe-Litwa et des secteurs Bambelota, Likombe, Luete. D'après les données du Recensement Scientifique de la Population du Zaïre en 1984, ces Topoke ont constitué 52,38 \% (128.613/245.548 habitants) de la population de la Zone d'Isangi, contre respectivement 15,89\% (128.613/809.266 habitants) et 2,98 \% (128.613/4.314.672 habitants) de la population de la Sous-Région de la Tshopo et de la Région du Haut-Zaïre (République du Zaïre, 1991 : 57-65). Un second groupe de Topoke est localisé dans la Collectivité-secteur de 
Yalikandja-Yanonge, créée en application du décret du 5 décembre 1933 sur les circonscriptions indigènes (chefferies et secteurs). Il s'agit des Topoke ayant constitué l'ancienne chefferie de «LIKOLO » et rattachée, de même que les chefferies de MaringaLopori, de Bambole et de Bakusu, au secteur ainsi créé (Bilusa, 1980 : 28). Ce groupe, appelé communément celui des Topoke de "LIKOLO", est une conséquence de mouvements de populations occasionnés par la pénétration des esclavagistes arabisés chez les Topoke.

Quelques familles topoke sont aussi localisées dans le bloc mongo (Bondombe, dans le bassin de la Tshuapa) de l'Equateur. Elles sont issues des Topoke qui ont fui les expéditions punitives conduites par M. Pimpurnaux, ancien commissaire du district de l'Aruwimi, en 1905, à la suite du massacre de deux agents blancs de la Compagnie du Lomami par les guerriers de Yaboila (Bolamba, 1975).

9 Rappelons que depuis l'installation de cette Compagnie chez les Topoke en $1899^{1}$, le mauvais traitement des populations lors de la récolte du caoutchouc naturel (celui des lianes) était un fait habituel. Ce qui finit par engendrer un incident entre les Topoke et les agents blancs de cette compagnie.

10 Un jour, en effet, Botuma bo Boono, ancien chef du village Yaboila (clan topoke de Bambelota), se disputa avec son épouse Limbaya et finit par la frapper. La femme, très touchée, dit à son mari : «Inde Toende A Ndwa, Ati Baumba Bafafonde », littéralement «si ce soleil est réellement ardent, pourquoi pourrissent alors nos légumes ». Les femmes de Yaboila reprochèrent, par cette expression, aux hommes de s'être montrés longtemps impuissants face à l'oppression dont l'ensemble de la société topoke était victime.

11 Blessés dans leur orgueil, Botuma bo Boono et son jeune frère Bosongo, en compagnie d'autres guerriers du village, firent irruption chez les deux agents blancs de la Compagnie $\mathrm{du}$ Lomami, les tuèrent et les mangèrent (Bolamba, 1975). Ils tatouèrent, note Walle, "le plus jeune et le renvoyèrent plus tard à Basoko (chef-lieu de District d'Aruwimi)" (Walle, 1981 : 21). Les deux agents de la compagnie s'appellaient Lhoor et Ruette.

12 Les itinéraires migratoires des Topoke restent encore peu connus. Pour Moeller, "les Topoke déclarent occuper leurs terres ancestrales et ne rien connaître de leurs migrations" (Moeller, 1936 : 197). Deux faits semblent justifier cette constatation : d'abord le manque des données, ensuite l'attachement des Topoke à leurs terres ("HUMA»), clairement exprimé par cette chanson :

\begin{tabular}{|l|l|}
\hline Ago gaison ane & (c'est ici chez nous) \\
\hline Ago gaiso ane & (c'est ici chez nous) \\
\hline Aobeel'esu mbeo la kes & (où nous traitons nos affaires) \\
\hline
\end{tabular}

Pour De Book, administrateur territorial d'Isangi, l'origine des Topoke se situerait vers le Nord (bassin de l'Ubangi-Uele), qu'ils auraient abandonné devant les menaces des Baboa, poussés eux aussi par les Zande. Dans leur marche vers le Sud, ils se seraient d'abord installés sur la rive droite du fleuve Zaïre (vers le bas Aruwimi). De là, ils traversèrent ce fleuve pour s'établir dans l'angle formé par le Lomami et le fleuve Zaïre, ayant ainsi repoussé les premiers occupants (Bambole et Bangando) plus au Sud (Betau, 1973: 12). Cette version est reconnue par les traditions lignagères et villageoises ${ }^{2}$. 


\section{Des concepts "ESO" et "Topoke"}

Ces deux termes sont l'objet de plusieurs controverses. Nous essayerons d'en esquisser quelques principaux traits.

En 1903, dans son ouvrage intitulé "The Curse of Central Africa", le capitaine Guy Burrows parle de Topoke, essentiellement guerriers. On y voit un chef topoke entouré de 22 crânes de ses victimes (Burrows, 1903: 86-87). Cette même appellation est aussi employée par Harry Johnston en 1922. Le Topoke, dit-il, est une langue. Il la classe dans le groupe des langues bantoues de l'entre Aruwimi-Congo-Lomami (Johnston, 1922 : 509-519).

6 Torday et Joyce, de leur côté, parlent de "Tofoke", gouvernés par les chefs des villages. Au début, disent-ils,

"toute la tribu était sous l'autorité d'un seul chef, qui plaçait ses parents comme gouverneurs des principaux villages. Ce sont les descendants de ces derniers qui gouvernent actuellement. Ils ne sont pas tout à fait indépendants et reconnaissent la suzeraineté d'un chef descendant de l'ancien grand chef et qui se nomme Lobela" (Torday \& Joyce, $1922: 201$ ).

Contrairement à ce qu'affirment ces deux ethnologues, les Topoke n'ont pas connu de pouvoir politique centralisé. Le pouvoir s'est limité aux familles au sein desquelles les guerriers ont joué un rôle de premier ordre car ils garantissaient la paix dans la contrée. A l'époque de la pénétration arabisée et plus tard lors de l'occupation européenne, les envahisseurs firent face à la résistance farouche des Topoke, dirigés par leurs principaux guerriers. Parmi ces derniers, on peut citer Lobela, issu du clan Kombe, proche d'Isangi ${ }^{3}$.

En 1948, le Révérend Père Hulstaert les appelle Topoke et leur langue GESO ou ESO. Il relativise néanmoins ses vues en soulignant que cette langue est peu connue (Hulstaert, 1948 : 23). Guthrie, dans sa classification, place la langue des Topoke dans la zone C., groupe 50. Il parle de Puki, tu (Topoke), classée C53 (Guthrie, 1948 : 75).

(Van Bulck, 1950 :34) les éléments fournis deux années auparavant par le Révérend Père Hulstaert, formule une nouvelle problématique sur l'orthographe des noms ethniques du Congo belge. Il écrit ceci :

"Les noms des tribus et des langues, même alors que nous sommes renseignés sur leur forme exacte, sont néanmoins orthographiés de façon les plus diverses. Certaines variantes sont à mettre sur le compte de simples différences de transcription, d'autres sont dues à la variabilité des noms eux-mêmes dans la langue en question (ajout ou permutation des préfixes ou des suffixes). Y a-t-il une solution possible qui, tout en restant simple et à la portée de tous, permette d'éviter cette confusion ?" (Van Bulck, 1954 : 5).

Il suggère plusieurs solutions parmi lesquelles nous épinglons les suivantes :

$1^{\circ}$ les noms ethniques sont pourvus de leur élément grammatical distinctif préfixé ou suffixé :

\section{ba Topoke,qui signifierait les Topoke ou de Topoke.}

Faisons remarquer que cette forme n'a de sens que dans la mesure où elle sert d'élément déterminatif : "bana ba Topoke» qui signifie "les enfants de Topoke". Cet élément (préfixe ba) ne permet cependant pas de fournir des renseignements sur la descendance. La version locale voudrait "Ya Topoke", c'est-à-dire issus de Topoke. D'une manière 
générale, les noms des villages, des familles ou des clans sont précédés du préfixe Ya. Voyons-en quelques exemples :

\begin{tabular}{|l|l|l|}
\hline - YA-Losuna & - YA-Basundu & -Ya-Liendja \\
\hline - YA-Boila & - YA-Baila & -YA-Isenge \\
\hline - Ya-Bongengo & - YA-Boololo & - YA-Osonga \\
\hline - Ya-Timbo & - Ya-Faga & - Ya-Longankja, etc. \\
\hline
\end{tabular}

$2^{\circ}$ Les noms ethniques sont donnés au singulier et au pluriel.

Concernant les Topoke, il aboutit à ceci :

\begin{tabular}{|l|l|}
\hline Singulier & Pluriel \\
\hline Tofoke & Topoke \\
\hline Topoke & Topoke \\
\hline Tupuki & Topoke \\
\hline
\end{tabular}

Cette série de solutions appelle plusieurs éléments de critique :

1. parmi les trois règles définies ci-haut, seule la deuxième correspond à la dénomination actuelle des Topoke ;

2. du point de vue de la linguistique, leur appellation serait "Tofoke", puisqu'il n'existe pas de lettre $\mathbf{P}$ dans le système phonétique topoke ;

3. Tupuki n'a aucune signification dans la culture topoke ;

4. du point de vue grammatical, les deux formes (singulier et pluriel) seraient les suivantes :

\section{I-FOKE TO-FOKE}

Ce qui est conforme à la règle générale, car les noms commençant par I ont leur pluriel en TO :

\section{Exemples:}

- I-tanga, pluriel to-tanga (un petit filet, des petits filets);

- I-sandu, pluriel to-sandu (un arbres, des arbres).

Et même lorsqu'il s'agit des exceptions, l'usage ne correspond nullement à celui qui est proposé par Van Bulck car, dans ce cas, les noms commençant par I ont leur pluriel en ba :

Un exemple :

I-Lomi, au pluriel ba-lomi (un sein, des seins).

$3^{\circ}$ Van Bulck fournit ensuite une liste de cinq colonnes. Seules les quatre premières nous intéressent, la dernière n'étant qu'un élément de détermination. Ces quatre colonnes sont les suivantes :

- col. 1 : le nom ethnique simplifié ; 
- col. 2 : le nom ethnique scientifique au pluriel ;

- col. 3 : le nom ethnique scientifique au singulier ;

- col. 4 : le nom linguistique scientifique.

Au sujet des Topoke, cette règle s'applique comme suit :

\begin{tabular}{|l|l|l|l|}
\hline 1 : Topoke & $2:$ ba Topoke & $3:$ ga Topoke & $4:$ Topoke \\
\hline
\end{tabular}

Les critiques formulées à la rubrique précédente s'appliquent aussi à cette série. Van Bulck distingue même, dans son étude, les Liutua des Topoke, alors qu'ils font partie intégrante de ces derniers.

$364^{\circ}$ Il nous fournit une liste à 2 colonnes dont la première concerne le nom ethnique d'usage courant, tandis que la seconde se rapporte à celle qu'il considère comme la nomenclature scientifique de référence. On y voit ceci sur les Topoke :

\begin{tabular}{|l|l|}
\hline tofoke & topoke \\
\hline topoke & tupuki. \\
\hline
\end{tabular}

$375^{\circ}$ Enfin, dans la dernière liste de son étude, il répertorie les groupements ethniques $d u$ Congo belge étudiés. Les Topoke y sont notés « Topoke».

Enfin, Bryan parle de Poke (Topoke) ou Tofoke dont le nom de la langue se confond avec celui du groupe ethnique. Parmi les sous-groupes de cette langue, il distingue les suivants :

- Topoke

- Liutua

- Baluolambila, probablement d'origine non topoke (selon lui)

- (A) Lomboki (pseudo-lokele)

- Likolo (Bryan, 1959 : 46-47).

Cette dernière ne permet guère de faire progresser nos connaissances sur ce peuple. D'abord le Topoke ne peut pas être considéré comme un sous groupe. Bryan voudrait-il entendre par là certains clans topoke mal définis ? Ensuite les Liutua, les Baluolambila, les Bolomboki et les Likolo appartiennent à la grande famille topoke.

Que s'est-il passé ? Pourquoi cet éventail d'orthographes et d'appellations? Les travaux existants offrent peu d'éclairage à cette question. Selon que l'on se réfère à tel ou tel devancier, on adopte sa terminologie, en la remaniant peut-être, sans que des justifications soient fournies sur l'état des investigations ayant abouti à tel ou tel résultat. C'est peut-être à ce genre de difficultés que fut confronté Burssens qui inclut les Topoke dans le groupe lokele (Burssens, $1954: 21$ ).

41 Les concepts «ESO » et «Topoke» recouvrent plusieurs significations. Etudions-les séparément avant de préciser l'appellation qui est acceptée localement. 


\section{$1^{\circ}$ Le concept ESO} des chenilles appelées "SOGO". Ainsi qu'on l'a fait pour le cas précédent, procédons par la même démonstration :

\begin{tabular}{|l|l|l|}
\hline Groupe ethnique & Arbre & Chenilles \\
\hline Topoke & OSOGO & SOGO \\
\hline Lokele & OSOO & SOO \\
\hline Bambole & OSOHO & SOHO \\
\hline Bangando & OSOHO & SOHO \\
\hline
\end{tabular}

Plusieurs hypothèses essaient d'expliquer les origines du mot ESO attribué aux Topoke. Nous en avons retenu, dans cet exposé, quatre:

(a) « ESO » proviendrait de « ESU » signifiant "nous". ESU est le pluriel de « ELI » (c'est-àdire Moi). est aussi utilisé par les voisins des topoke (Bambole, Bangando, Lokele, Turumbu) :

\begin{tabular}{|l|l|l|}
\hline Groupe ethnique & Concept ESU & Signification \\
\hline Topoke & ESU & Nous \\
\hline \hline Bambole & ESU & Nous \\
\hline Bangando & ESU & Nous \\
\hline Lokele & ISO & Nous \\
\hline
\end{tabular}

Par conséquent, tous ces groupes seraient aussi désignés par ce terme.

b) "ESO" signifierait les gens qui grimpent sur l'arbre appelé "OSOGO" pour y ramasser

Faisons remarquer que cette pratique est courante dans tous ces groupes ethniques, à l'exception peut-être des Lokele qui sont riverains.

c) "ESO" proviendrait de "GESGO", qui voudrait dire chaleur, par référence à l'esprit "belliqueux" des Topoke. Ici aussi, les données linguistiques montrent que cette hypothèse ne satisfait pas la réflexion, ainsi qu'on peut le constater à travers les éléments de comparaisons ci-après :

\begin{tabular}{|l|l|}
\hline Topoke : & GESOGO \\
\hline \hline Lokele : & ESOO \\
\hline
\end{tabular}




\begin{tabular}{|l|l|}
\hline Bambole : & ESOHO \\
\hline Bangando : & ESOHO \\
\hline
\end{tabular}

Si, dans l'ensemble, le même terme se rencontre dans tous ces groupes ethniques voisins et renferment la même signification, le seul élément de différenciation se situerait dans "l'état d'esprit" de ces populations. Les Topoke sont reconnus guerriers et turbulents, par rapport à leurs voisins.

50 d) "ESO" signifierait "gens de l'intérieur", "qui habitent la forêt", par opposition aux riverains appelés "LIANDE". On rapporte que lorsque les Européens arrivèrent pour la première fois dans les terres d'Isangi, ils entrèrent d'abord en contact avec les populations riveraines (donc les Lokele). Ils demandèrent alors à ces dernières s'il y avait d'autres habitants dans l'épais manteau de forêt. Les Lokele répondirent "ESO". Ce dernier terme finit par être retenu comme celui qui désignerait les Topoke.

\section{$2^{\circ}$ Le concept "TOPOKE"}

Une seule hypothèse existe de nos jours sur l'origine du nom "topoke". Ce concept serait une déformation de "Tofoe", signifiant "nous ne comprenons pas". "Tofoe" est la négation de "Toendoa", c'est-à-dire "nous saisissons", "nous comprenons", du verbe à l'infinitif "OOI" ou "OOA", signifiant "comprendre, saisir". On rapporte que lorsque les premiers colonisateurs s'étaient adressés, à leur tour, aux Topoke pour connaître leur nom, ces derniers répondirent "Tofoe". Cette réponse, résultant de l'incompréhension entre les deux parties en présence, serait dès lors adoptée pour désigner les Topoke.

La confrontation de toutes ces hypothèses avec les éléments tirés de l'histoire locale nous permet de soutenir que "ESO" est effectivement le nom renseigné par les Lokele aux premiers agents européens. Et de là se développa toute une attitude de mépris et d'hostilité entre les Topoke et les Lokele, les derniers considérant les premiers comme des gens de classe inférieure. Pour les Topoke, "ESO" n'est pas leur vrai nom. "ESO" est rejeté parce qu'il est l'une des manifestations du mépris des Lokele à l'égard des Topoke. L'expression "TOTOTO TWA ESO", c'est-à-dire "les petites gens de ESO" courante chez les Lokele semble être le reflet d'un antagonisme permanent entre ces deux groupes ethniques. "ESO" renferme donc une connotation péjorative et constitue une injure à l'égard des populations que nos appelions "Topoke". C'est ce dernier terme (Topoke) qui est accepté.

\section{Perspectives d'avenir}

Les Topoke constituent l'une des sociétés qui n'a pas encore fait l'objet d'investigation scientifique sérieuse. Un effort est donc à accomplir, par exemple dans le domaine de l'histoire, en vue d'établir l'identité de ces populations. Dans cet élan, il importera de réfléchir sur l'antériorité ou la postériorité d'une appellation par rapport à l'autre.

Pendant nos investigations menées en 1979, certains Topoke nous avaient déclaré que leurs noms seraient "Libelase", c'est-à-dire "qui a le pouvoir de procréer". Dans leurs justifications, ils avaient fait allusion à l'importance des Topoke en matière de 
descendance, par rapport aux autres enfants d'IONGYA-ONGYA, ancêtre fondateur des Topoke et des Lokele. Cette explication ne nous a pas satisfait car le fait d'avoir une nombreuse descendance est postérieur au nom que l'on donne à une personne lors de sa naissance.

S'il est aujourd'hui admis que "ESO" n'est pas le nom des Topoke, les données sont loin d'être fixées de manière définitive car, avant de répondre "Tofoe", ces populations avaient déjà un nom. C'est celui-là qu'il importera de chercher à déterminer.

\section{BIBLIOGRAPHIE}

BETAU NG. B., L'impact des conflits ethniques sur le pouvoir politique dans la Zone d'Isangi (Haut-Zaïre) : 1957-1964, Lubumbashi, UNAZA, Campus de Lubumbashi, mémoire de licence en Sciences politiques et administratives, 1973 (inédit).

BIEBUYCK, D., "Note sur les recherches anthropologiques et ethnologiques urgentes au Congo Belge", extrait du Bulletin of International Commit tee on Urgent Anthropological and Ethnological Research, $\mathrm{N}^{\circ} 3,1960$.

BILUSA, B.B., Histoire de la Population de la Zone d'Isangi (Haut-Zaïre), 1932-1973, Lubumbashi, UNAZA, Campus de Lubumbashi, mémoire de licence en Histoire, 1980, (inédit).

BOLAMBA bo Yaya, Les Topoke face a la domination étrangère et leur rôle dans les mouvements de décolonisation, Lubumbashi, UNAZA, Campus de Lubumbashi, mémoire de licence en Sciences politiques et administratives, 1975 (inédit).

BRYAN, M.A., The Bantu Languages of Africa, London/New York/Cape town, Oxford University Press, 1959.

BURROWS, G., The Curse of Central Africa, London, R.A. Everett \& Cie Ltd, 1903.

BURSSENS, A., Introduction h l'étude des langues bantoues du Congo Belge, Anvers, Ministère des colonies, Ecole Coloniale, 1954.

GUTHRIE, M., The Classification of the Bantu Languages, London/New York/Toronto, Oxford University Press, 1948.

HUSLTAERT, G., Carte linguistique du Congo Belge, Bruxelles, IRCB, 1948.

JOHNSTON, H., A Comparative Study of the Bantu and Semi-Bantu Languages, Vol. II, Oxford, Clarendon Press, 1922.

MAES, J. \& BOONE, O., Les peuplades du Congo Belge, Bruxelles, Imprimeries Veuves-Monnon, 1935. MOELLER, A., Les grandes lignes des migrations des Bantous de la Province Orientale du Congo Belge, Bruxelles, IRCB, 1936.

Mouvement Géographique, n 18, 1899, p. 218.

REPUBLIQUE DU ZAIRE, Ministère du Plan et Aménagement du Territoire, Zaïre, Recensement scientifique de la population. Juillet 1984. Totaux définitifs, Kinshasa, INS, 1991. 
TORDAY, E. \& JOYCE, T.A. (M.A.), Notes ethnographiques sur des populations habitant les bassins du Kasai et du Kwango Oriental. 1. Peuplades de la forêt. 2. Peuplades des prairies, Bruxelles, Annales du Musée du Congo Belge, 1922.

Van BULCK, G., Les deux cartes linguistiques du Congo Belge, Bruxelles, IRCB, 1950.

Id., Orthographie des noms ethniques du Congo Belge, Bruxelles, IRCB, 1954.

WALLE, S.B., "L'histoire politique des Topoke à Kisangani (Haut-Zaïre), des origines à 1964", in Les Cahier du CEDAF, $n^{\circ}$ 3, Bruxelles, 1981.

\section{NOTES}

1. Cette compagnie fut créée par le décret du 5 juillet 1898 au capital de 3.000 .000 frs et avait son siège social à Bruxelles. Son premier directeur, le lieutenant Lemery, accompagné de ses deux adjoints, MM. Blampain et Sterkx, arriva le 14 février 1899 à Ilambi (village topoke du clan Kombe) sur la Lomami et y érigea le premier poste de la compagnie. Cfr Mouvement Géographique, $n^{\circ} 18,1899$, p. 218.

2. Données d'enquête orale, recueillies à Tongombe, le 30/8/1979. Citées par Bilusa (1980:129).

3. Une étude sur les mutations socio-politiques observées dans la société topoke depuis le XIX ${ }^{\mathrm{e}}$ siècle est actuellement en cours de réalisation.

\section{RÉSUMÉS}

Today, as a result of the numerous works on the matter, we know about the effects of European penetration on the organization of African societies. We can hold up as an example the emergence of a new political map of Africa where ethnic groups are scattered over various territories and, on an economic level, Africa's insertion into a system unsuited to its basic structures.

However, there is one subject that has not attracted the attention of historians until now. It concerns the naming of ethnic groups which nowadays live in this part of our planet. Most of these ethnic groups are known under various names. Even worse, some of them are known by nicknames. These few lines concern the naming of the TOPOKE of High Zairre which are also known in written litterature as the ESO, GESO, GESOGO, GESOHO, TOPOKE, TUPUKI, TOFOKE, TUFUKI.

\section{AUTEUR}

\section{BILUSA BAILA BOINGAOLI}

Assistant à la Faculté des Sciences Sociales, Administratives et Politiques, Université de Kisangani, B.P. 2012, Haut-Zaïre. 